This item was submitted to Loughborough's Research Repository by the author.

Items in Figshare are protected by copyright, with all rights reserved, unless otherwise indicated.

\title{
Heat generation and temperature distribution in DC metallized polymer film
}

\section{capacitors}

PLEASE CITE THE PUBLISHED VERSION

PUBLISHER

(c) IEEE

VERSION

VoR (Version of Record)

LICENCE

CC BY-NC-ND 4.0

\section{REPOSITORY RECORD}

Lee, Yuen-Pen, and Michael G. Kong. 2019. "Heat Generation and Temperature Distribution in DC Metallized Polymer Film Capacitors". figshare. https://hdl.handle.net/2134/5231. 
This item was submitted to Loughborough's Institutional Repository (https://dspace.lboro.ac.uk/) by the author and is made available under the following Creative Commons Licence conditions.

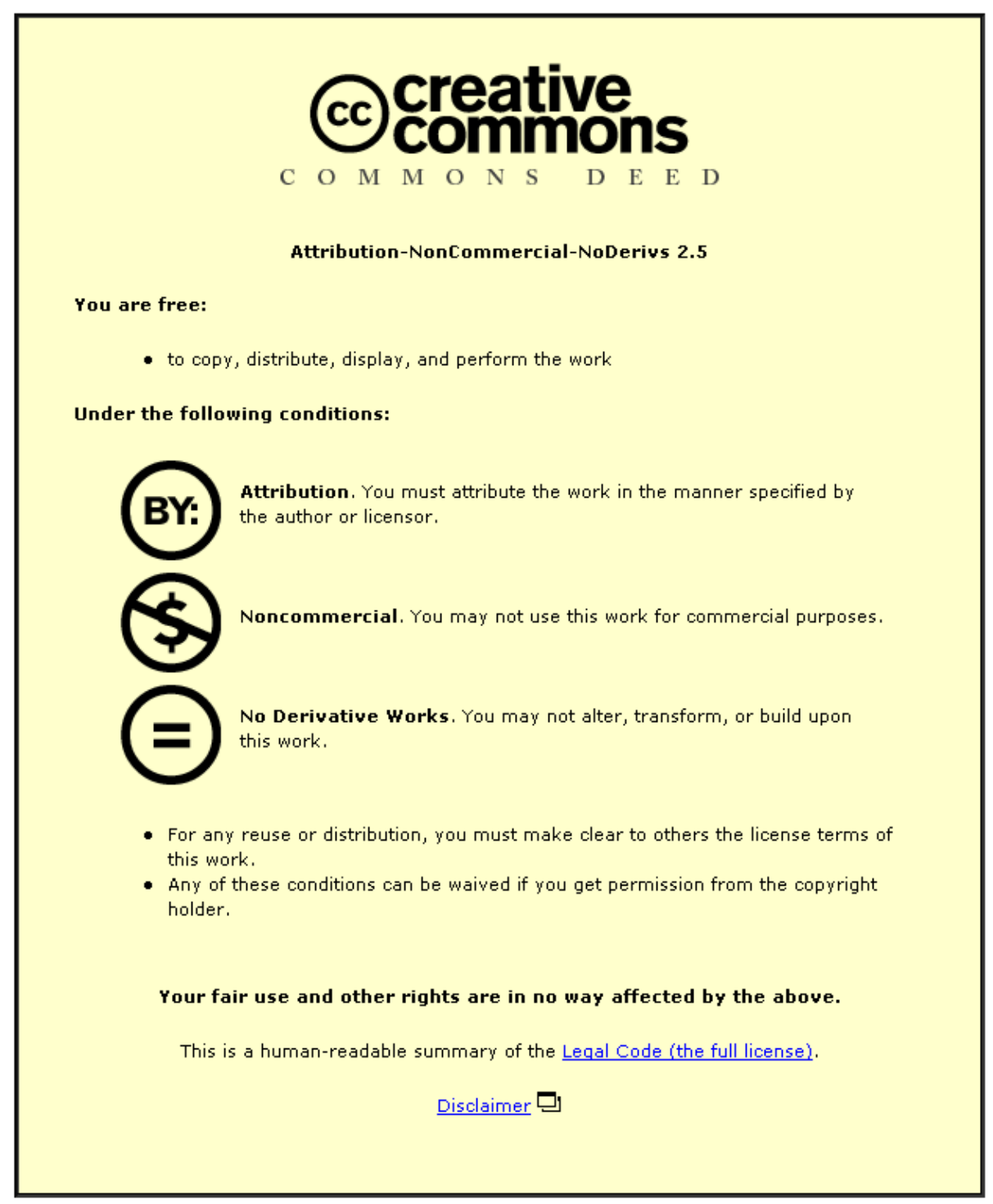

For the full text of this licence, please go to: http://creativecommons.org/licenses/by-nc-nd/2.5/ 
2001 Annual Report Conference on Electrical Insulation and Dielectric Phenomena

\title{
Heat Generation and Temperature Distribution in DC Metallized Polymer Film Capacitors
}

\author{
Y P Lee and M G Kong \\ Department of Electronic and Electrical Engineering \\ Loughborough University, Leicestershire LEII 3TU, UK
}

\begin{abstract}
It is known that elevated temperature can considerably reduce life expectancy of metallized polymer film capacitors. For film capacitors in service, both application of a time-varying external voltage and the extinction of internal breakdown events can deposit significant heat onto the capacitor structure leading to an undesirable temperature rise. Often such heat generation is also localized spatially and the resulting temperature gradient enhances the probability of subsequent breakdowns. To gain an understanding, a numerical model is developed to simulate the dynamics of heat generation and transfer under the switching impact of an external DC voltage. Thermal processes in solids (polymer layers, electrode coating, and zinc-spray segment) are modelled with conduction mechanism, whereas convection mechanism is considered for silicone oil and air. Heat generation is induced by ohmic losses of the externally applied electric field, which can be calculated from an equivalent circuit model recently developed for metallized film capacitors. Thus the heat transfer model interfaces with the equivalent circuit based field calculation model. Based this electrothermal model, numerical examples are used to study temperature distribution within film capacitors and then to examine the likelihood of localized temperature hot-spots that may lead to spatially preferential breakdowns.
\end{abstract}

\section{Introduction:}

There has been significant progress in the technology of metallised polymeric film capacitors over the past 20 years[1][2]. One of main drivers in the field is higher energy density with increasingly reliability. Recent innovations in ageing analyses are introduced for predicting capacitor performance and fault tolerance[3]. Thermal ageing is one of main failure mechanisms in polymeric film capacitors[2]. In the $40-65^{\circ} \mathrm{C}$ range, experimental data in [3] shows that the life of the metallized film capacitor is decreased by a factor of 2 for every $8^{\circ} \mathrm{C}$ of temperature increase. This highlights the need to gain a thorough understanding of heat generation and temperature distribution for the film capacitor performance. In this paper, an electrothermal model is developed to study the evolution of temperature distribution in DC metallized polymer film capacitors under different heat generation conditions. Both heat conduction and convection mechanisms are considered. Heat generation is induced by ohmic losses of the externally applied electric field or the internally breakdown events. Numerical computations are then used to assess localized temperature hot-spots on the surface of the metallized films.

\section{Problem definition and its model:}

A DC metallized polymer film capacitor typically consists of a few thousand flattened polymer layers stacked together and immersed in an impregnation medium in the packaging case unit. As shown in figure 1 , each fattened polymer layer is coated on one side with thin metallic coating. The metallic coating has one of its side edges connected to an electrode terminal and other insulated with side margin. The side margin is filled up by the impregnation medium. Side margins are on alternating sides of the stacked polymer layers so that the metallic coatings are connected altematively to two external electrode terminals.

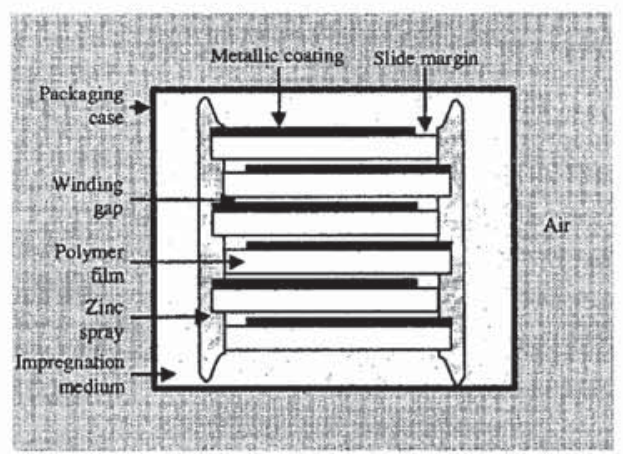

Figure 1: a section of a typical DC metallized polymer film capacitor.

As a first step, we consider a simplified one-layer film capacitor model to allow a one-dimensional simulation of the thermal evolution in film capacitors. Furthermore, we assume that the polymeric films on the top and bottom of the thin metallic coating and the impregnation medium in the winding gap are perfect thermal insulators. The metallic coating is connected to zinc-spray at both ends with the margin side filled up by impregnation medium. The far end of both zincsprays was interfaced with impregnation medium. We also assume that heat flow is directed outwards to air through the vertical plate of the packaging case at the far ends of both impregnation medium sections. The thermal model structure is illustrated in figure 2. Zinc is a good conduction material and the length of the zinc spray, $L_{2 n}$, is a variable depending on conditions of heat generation. For instance, $L_{z n}$ is equal to the thickness of the polymer layer if the heat generation is induced by externally applied electric field, in which case each layer will be assigned to an identical temperature distribution. Consequently the thermal potential difference for all the metallic layers connected to the same zinc-spray is the same. The heat path for the zinc spray entirely depends on the distance between the adjacent metallic layers. On the other hand, $L_{z n}$ is equal to the thickness of the capacitor unit if the heat generation is induced from internal breakdown discharges. 


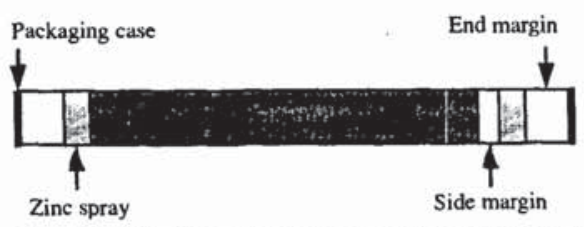

Figure 2: A simplified single-layer film capacitor of thermal model.

The impregnation medium is assumed as silicone oil, and temperature-dependent properties of all materials used in film capacitors are assumed unchanged in the timescale considered here. This assumption is reasonable as shown in the stability test to International Electrotechnique Commission (IEC) requirements[4]. The maximum temperature change in the internal and external capacitor unit under the stability test was below $10^{\circ} \mathrm{C}$. A temperature change of $10^{\circ} \mathrm{C}$ in the range from 20 to $70^{\circ} \mathrm{C}$ does not alter significantly the material properties used in film capacitors [6]. Ohmic losses induced heat generation can be calculated from an equivalent circuit model recently developed for metallized film capacitors[5]. This facilitates an interface between the heat transfer model and the equivalent circuit based field calculation model.

Heat transfer processes can be classified into three basic types, namely conduction, convection and radiation [6]. The radiation energy exchange between two surfaces of finite size area, A, is given by [7]

$$
q=A h_{r}\left(T_{1}-T_{2}\right)
$$

where $h_{r}=4 \varepsilon \sigma T_{m}^{3}$ is called the radiation heat transfer coefficient. $\sigma$ and $\varepsilon$ is the Stefan-Boltzmann constant and emittance of the surface respectively. $T_{1}$ and $T_{2}$ is temperature for two surface areas. $T_{m}$ is the mean of $T_{1}$ and $T_{2}$. Thermal resistance is defined as the total heat flow thought a unit with a thermal potential difference. For conduction, the thermal resistance is given by [6]

$$
R_{k}=\frac{\Delta x}{k A}
$$

where $k$ is the thermal conductivity of a unit material with a distance, $\Delta x$ and a cross section area, $A$. For convection, the thermal resistance is defined as [6]

$$
R_{c}=\frac{1}{h_{r} A} \text {. }
$$

Consider a cubic volume material with the side length of $1 \mathrm{~m}$, which has two opposite surfaces with temperature equal to $310 \mathrm{~K}$ and $300 \mathrm{~K}$ respectively. The thermal resistance due to conduction and radiation can be estimated using eqs. (1), (2) and (3), and the results are listed in table 1 . The thermal resistance calculation for the heat radiation is based on a blackbody assumption that $\varepsilon_{r}$ is equal to 1 . The properties of all materials used in film capacitors at ambient temperature of $20^{\circ} \mathrm{C}$ are based on data in [6][8].

\begin{tabular}{|c|c|c|c|c|}
\hline & \multicolumn{3}{|c|}{ Conduction } & Radiation \\
\hline Material & $\mathrm{Al}$ & $\mathrm{Zn}$ & $\mathrm{PP}$ & Blackbody \\
\hline $\mathrm{R}(\mathrm{K} / \mathrm{W})$ & 0.00438 & 0.00892 & 6.25 & 0.1554 \\
\hline
\end{tabular}

Table 1 Thermal resistance by heat conduction and radiation for different materials.
The thermal resistance calculation for the heat radiation is an overestimate because of the blackbody assumption. In practice heat transfer by conduction for polypropylene (PP) and by radiation are likely to be negligible compared with the heat conduction in metallic sections. Based on the empirical relations for free convection in [8] and data in $[6][8]$, the heat transfer processes by the free convection mechanism for the silicone oil and air in the typical film capacitor is also negligible. As a result, we only consider the heat conduction mechanism in our thermal model. Based on the above assumptions, the thermal model is illustrated in figure $3 \mathrm{a}$ where the solid arrow indicates the heat flow in this one-dimensional thermal model. The more realistic heat flow should be that indicated by the dash arrows in figure $3 \mathrm{a}$. This allows us to modify our thermal model to allow precise heat flow as shown in figure $3 \mathrm{~b}$ in which the zinc spray section extended from electrode layer are bent by $90^{\circ}$ to allow heat flow in one-dimension fashion.

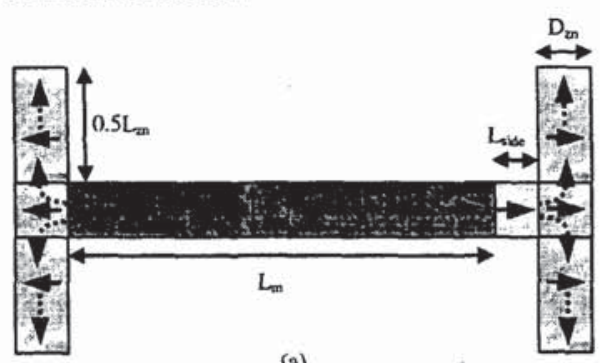

(a)

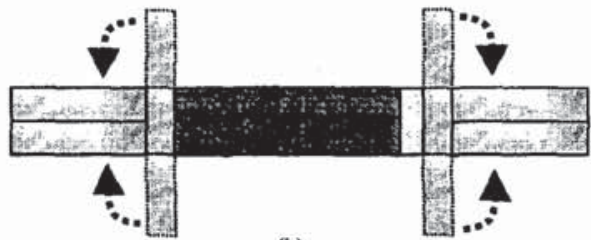

(b)

Figure 3: A simplified thermal model for film capacitor with (a) an ideal; and (b) a simplified capacitor structure.

If $j$ denotes all neighbouring nodes connected to node $i$ and $q_{i}$ denotes the rate of heat addition (external or internal) to the node, the future temperature of node $i$ is determined by [6]

$$
T_{i}^{\cdot}=T_{i}+\delta t\left(\sum_{j} \frac{T_{j}-T_{i}}{R_{i j} C_{i}}+\frac{q_{i}}{C_{i}}\right)
$$

where

$$
R_{i j}=\frac{\delta_{i j}}{k A_{i j}} \text {, for conduction and } C_{i}=V_{i} \rho c_{p} .
$$

These equations express the temperature at a given node in terms of discrete spatial steps $\left(\delta_{i j}\right)$ and discrete time steps $(\delta t) . R_{i j}$ is the total thermal resistance of two nodes namely $i$ and $j . A_{i j}$ is the cross section area of the unit with a spacing $\delta_{i j}$. $C_{i}$ and $V_{i}$ are the thermal capacitance and the volume of the lump volume surrounding the node i. $\rho$ and $c_{p}$ are respectively the 
mass density and the specific heat of the unit volume at node $\mathrm{i}$.

\section{Results with DC stresses:}

Based on eq.(4) for the thermal model in figure $3 \mathrm{~b}$ and a computer program is subsequently developed to simulate the evolution of temperature distribution in the single film capacitor. The rate of heat, $q_{i}$, in eq.(4) is calculated from an equivalent circuit model. For numerical examples discussed here, the length of the metallic layer $\left(L_{m}\right)$ and side margin $\left(L_{\text {side }}\right)$ are $96 \mathrm{~mm}$ and $4 \mathrm{~mm}$ respectively. The thickness of the zinc-spray $\left(D_{z n}\right)$ and metallic layer $\left(D_{m}\right)$ are $2 \mathrm{~mm}$ and $10 \mathrm{~nm}$ respectively. The length of the zinc spray is set to the thickness of the polymer film at $7.4 \mu \mathrm{m}$. The surface resistance of the metallic coating is set to $25 \Omega$. Based on the above parameters, the time constant for the equivalent circuit model is about $1.38 \mu$ s[5]. The charging time is a small fraction of the discrete time step used in the thermal model. Therefore, the initial temperature distribution of the thermal model was an accumulated result of heat generate from the DC charging process. Furthermore, we assume no additional external or internal heat generation in the thermal model during the transient processes.

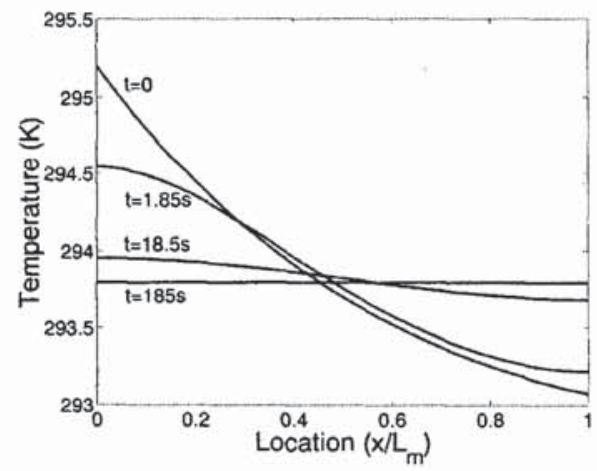

Figure 4: Spatial distribution of temperature in metallic layer at difference time steps.

Figure 4 is a plot of the spatial distribution of temperature in metallic layer at different time steps. Initially the peak temperature occurs at the left-hand edge of the metallic layer through which the majority of electric energy is fed into the system. The peak temperature remains in this area during the transient process before the heat transfer process reaching a steady state at approximately $t=185 \mathrm{~s}$. As a result, the peak average temperature during the entire transient process occurs at the left-hand side of the metallic layer as shown in figure 5. The localized temperature hotspot for DC film capacitor is at the edge of the metallic layer connected to the zinc-spray. It is interesting to note that the localized temperature hot-spot can be found directly from the accumulated energy calculated from the equivalent circuit model of DC film capacitors.

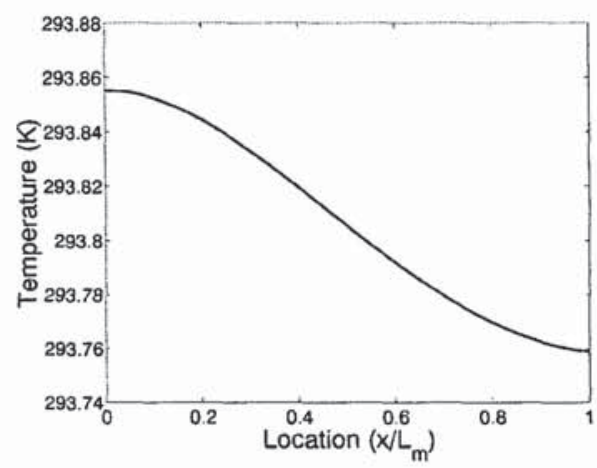

Figure 5 Distribution of the average temperature accumulated during the transient process.

\section{Results with internal breakdown events:}

When internal breakdown events extinguish in film capacitors, they deposit significant heat onto the capacitor structure. The heat generated depends on the characteristics of the breakdown events and their occurrence frequency. For cases studied here, we assume that the breakdown events result in a uniform temperature rise of $1 \mathrm{~K}$ across a metallic coating above the ambient temperature of the film capacitor. Furthermore, we assume the breakdown events are induced in the central metallic layer of the film capacitor. Therefore, the length of the zinc-spray $\left(L_{2 n}\right)$ for this case is equal to the thickness of the film capacitor. For practical film capacitors, $L_{z n}=2-5 \mathrm{~cm}$, and the case discussed here we choose $L_{z n}=2 \mathrm{~cm}$.

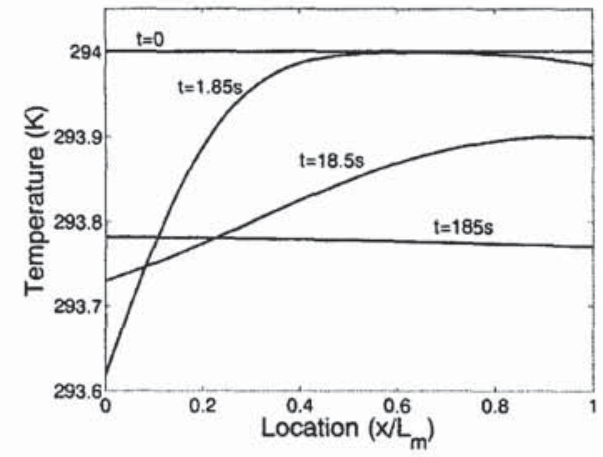

Figure 6 Spatial distribution of temperature in metallic layer at difference time steps for internal breakdown events.

Figure 6 is a plot of the spatial distribution of temperature on the metallic layer at different time steps for the heat generated by the internal breakdown events. The peak temperature now moves toward the right-hand side during the transient process before the heat transfer process reaches the steady state at $t=185 \mathrm{~s}$. As a result, the peak average temperature accumulated during the entire transient process is at the right-hand side of the metallic layer as shown in figure 7. It is interesting to note that $L_{2 n}$ mainly depends on the breakdown events. For instance, if breakdowns occur on each metallic layer with the same spatial distribution, $L_{z n}$ would be 
equal to the distance between the two adjacent metallic layers. For comparison, the average temperature accumulated for $L_{z n}$ being between $0 \mathrm{~mm}$ and $40 \mathrm{~mm}$ is calculated and shown in figure 7 . The results shown that the peak of the localized temperature hot-spot induced by breakdown events is decreased as $L_{z n}$ increases. Nevertheless, the difference between the peak and the lowest temperature becomes larger. It is clear that zinc-spray reacts as a heat sink for heat generated by the extinction of internal breakdown events. More importantly figure 7 also resembles the spatial distribution of broken fuses observed for striped down aged film capacitors. We believe that they are closely correlated and as such one key aging mechanism is the thermal effects of breakdown discharges. This correlation is also found useful to predict the life expectancy of practical film capacitors.

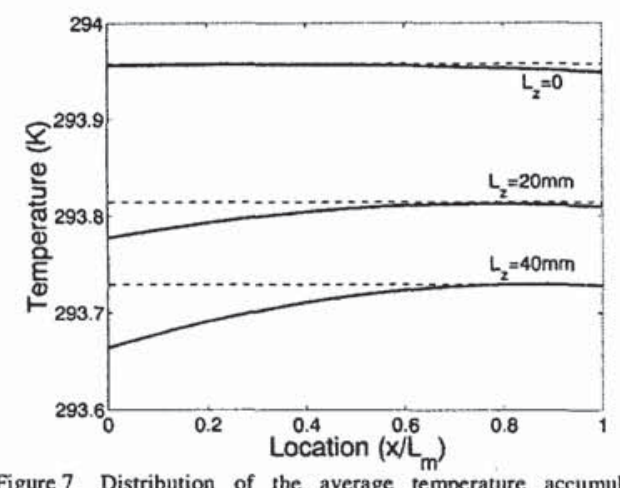

Figure 7 Distribution of the average temperature accumulated during the transient process at difference $L_{: n}$.

\section{Conclusion:}

An electrothermal model has been developed for metallized film capacitors. Based on a simplified film capacitor model, temperature distribution and localized temperature hot spot have been calculated for different heat generation conditions. It has been established that the localized hot-spots can be found from the accumulated energy for DC film capacitors during the charging processes. For heat generated by the extinction of internal breakdown event cases, the locations and temperature of hot-spots mainly depend on the features of the zinc spray and breakdown events.

\section{Acknowledgement:}

YPL acknowledges financial support from ABB Power T \& D, Capacitor Division, UK, and Loughborough University.

\section{Reference:}

[1] D. G. Shaw, S. W. Cichanowski, and A. Yializis, "A changing capacitor technology - failure mechanism and design innovations", IEEE Trans Electrical Insulation, vol.16, pp. $399-413,1981$.

[2] C. W. Reed and S. W. Chichanowski, "The fundamentals of ageing in HV power film capacitors", IEEE Trans Dielectric \& Electrical Insulation, vol.1, pp. 904-922, 1994.

[3] W. J. Sarjeant, "Capacitors", IEEE Trans Plasma Science, vol.26, pp. 1368-1392, 1998.

[4] International Electrotechnical Commission: Power electronics capacitors , CEI/IEC 1071-1, 1991.

[5] Y. P. Lee, M. G. Kong, M. R. Dunn, and D YoungCannon, "Temporal evolution of electric field distribution and energy dissipation in a metallized polymer film capacitor under dc, ac, or nonsinusoidal voltage stresses", presented at the 2000 IEEE International Conference on Dielectrics and Electrical Insulation Phenomena held in Victoria, BC, Canada, October 2000.

[6] A. J. Chapman, Heat transfer, third edition, MacMillan, New York, 1974.

[7] A. F. Mills, Heat transfer, second edition, PrenticeHall, New Jersey, 1999.

[8] J. P. Holman, Heat transfer, eighth edition, McGraw-Hill, New York, 1997. 